\title{
ENT Foreign Bodies: An Experience
}

\author{
Al Hussein Awad ${ }^{1}$ Mostafa ElTaher ${ }^{1}$ \\ ${ }^{1}$ Department of Otorhinolaryngology, Faculty of Medicine, Sohag \\ University, Sohag, Egypt \\ Int Arch Otorhinolaryngol 2018;22:146-151.
}

\begin{abstract}
Address for correspondence Al Hussein Awad, Department of Otorhinolaryngology, Faculty of Medicine, Sohag University, Sohag 82525, Egypt (e-mail: alhussein_awad@yahoo.com).
\end{abstract}

\begin{abstract}
Introduction Ear, nose and throat (ENT) foreign bodies (FBs) are common occurrences, particularly among children. The proper recognition, study, and management of FBs are required to prevent complications. Their consequences are greatly variable, from mild disturbances that may not require hospitalization up to life-threatening complications.

Objective To analyze the clinical spectrum of ENT FBs, the methods of removal, the outcomes and complications as seen in a tertiary referral hospital.

Methods This hospital-based cross-sectional retrospective study was performed from July 2014 to June 2016. Patients with any type of ENT FBs, regardless of age, were included in the study; data was collected from 1,013 patients (572 males and 440 females) with a mean age of 12.5 years.

Results Foreign bodies represented a large category among ENT emergencies (30\%). Children were affected more frequently, particularly $\leq 6$ years old. Swallowed FBs were the most common (53.6\%), followed by aural FBs (24.68\%), nasal FBs (19\%), and inhaled FBs (2.6\%). A total of $54.69 \%$ of ENT s were removed under general anesthesia (GA).

\section{Keywords}

- foreign bodies

- otorhinolaryngology

- emergency

Conclusion Foreign bodies (FB) in the ears, nose or throat are a common occurrence in otorhinolaryngology (ENT) emergency services. Children are the most affected age group. The commonest site of FB lodgment is in the throat. Ear, nose and throat FBs need to be properly managed to avoid complications.
\end{abstract}

\section{Introduction}

Foreign bodies (FBs) in the ear, nose, and throat (ENT) are commonly seen in the medical practice by otorhinolaryngologists, pediatricians, Emergency Department surgeons, physicians, and even in the primary care setting. ${ }^{1}$ Foreign bodies have been estimated to account for $\sim 11 \%$ of the cases seen in ENT emergency services. ${ }^{2-4}$ If not properly managed, they have a high potential for morbidity/mortality, and are costly to manage. ${ }^{5-7}$

Foreign bodies can be introduced spontaneously or accidently in both adults and children. Generally, ENT FBs are more common in younger children; this may be due to various factors, such as curiosity to explore orifices, imitation, boredom, playing, intellectual disabilities, insanity, and attention deficit hyperactivity disorder, along with the availability of the objects and absence of watchful caregivers. ${ }^{8}$

received

February 3, 2017

accepted

April 3, 2017

published online

July 14,2017

10.1055/s-0037-1603922. ISSN $1809-9777$.
Consequences of FB injuries vary from low impact disturbances with or without hospitalization to death. The variability is related to many factors, such as the chemical composition, shape and dimensions of the FBs, and the anatomical site involved. ${ }^{9}$

This study was performed to analyze FBs in terms of type, site, age, and gender distribution, method of removal, outcomes and complications.

\section{Methods}

A retrospective study was performed in the Department of ENT, Head and Neck Surgery in a tertiary referral hospital. The study population included all patients presenting with ENT FB lodgment in the Outpatient Department (OPD) or in the ENT emergency unit during the 2-year study period (from July 2014 to June 2016). The patients were evaluated carefully, with

Copyright @ 2018 by Thieme Revinter

Publicações Ltda, Rio de Janeiro, Brazil

License terms 
thorough history-taking and a complete ENT examination. Radiological investigations, like X-ray, were performed when the FB was not visible. This was followed by removal of the FB.

An anterior rhinoscopy was performed to diagnose nasal FBs. Rigid or flexible nasal endoscopic examinations were also performed in suspected cases of FBs in the nasal cavity that were not visualized with the anterior rhinoscopy. Direct vision with or without otoscopic assistance was employed to diagnose aural FBs. Examination under a microscope was an additional method for diagnosis, and it was useful for removal of the FB of the ear. For swallowed FBs, direct vision was obtained with a tongue depressor, indirect laryngoscopy was performed for co-operative patients, or transnasal flexible endoscopy. Plain X-rays of the neck were taken from patients with a history of FB ingestion. A rigid endoscopic examination (hypopharyngoscopy/ esophagoscopy) was performed in cases in which the FB was not visible in the X-ray to rule out presence of the FB or to determine its site of impaction, as well as to remove it. Rigid bronchoscopic removal of the FBs was performed for the patients who had radiographic evidence of an inhaled FB, and for those with strong clinical suspicion of an inhaled FB with no radiographic evidence.

Data were obtained from the ENT clinic registration books and ward admission record books. The following data were obtained from the patients: age, sex, type of FB and the site/side of impaction, complementary tests, duration of insertion, previous attempts at removal, methods of removal, outcomes, and complications. The patients were grouped according to the location of the FB into throat FBs (swallowed \& inhaled FBs), aural FBs, and nasal FBs. The data collected from the patients were analyzed using the Microsoft Excel 2010 (Microsoft, Redmond, WA, US) software. This study was conducted after receiving approval from the Research Ethics Committee of our institution.

\section{Results}

A total of 1,013 patients with ENT FBs were examined and managed at the ENT Department of a tertiary referral hospital during the period of July 2014 to June 2016. Foreign bodies represented a large category among ENT emergencies (30\% of all ENT emergencies). A total of 572 cases involved males (56.47\%) and 441 involved females (43.53\%).The age of the patients ranged from 1 to78 years, with a mean age of 12.5 years (mean \pm standard deviation $[S D]=12.5 \pm 14.2$ ). The major proportion of FB impactions (48.07\%) were found in children younger than 6 years of age, followed by children aged 7-12 years (28.33\%). None of the patients had psychiatric disorders or intellectual disabilities.

Among all of the FBs, swallowed FBs were the most commonly encountered (544 cases- $53.7 \%$ ), followed by aural FBs ( 250 cases- $24.68 \%$ ), nasal FBs (192 cases- $18.95 \%$ ), and inhaled FBs (27 cases- 2.67\%). - Fig. 1 and - Table 1 describe the distribution of ENT FBs by site, gender and age groups. The type of FB most commonly encountered differed according to the implicated anatomical site. The FBs were removed with or without local anesthesia (LA) in 411 (40.57\%) patients; 554 (54.69\%) patients required removal under general anesthesia (GA); and no FBs were found in 48 (4.74\%) patients, in spite of their positive history, as shown in - Table 2.

\section{Swallowed Foreign Bodies}

This group of patients was by far the most common, with 544 (53.7\%) patients presenting in the study period, of which 314 (57.7\%) were males and 230 (42.3\%) were females. A total of $62.9 \%$ of the patients who had come with swallowed FBs were children under the age of 6 years, and $16.5 \%$ were children aged 7- 12 years. The most common swallowed FBs were coins, which represented 410 (75.37\%) cases, followed by meat lumps in $32(5.88 \%)$ cases, fish bones in 30 (5.51\%) cases, pins in 22 (4.04\%) cases, chicken bones in 20 (3.68\%) cases, disc batteries in 14 (2.57\%) cases, and other FBs in 16 (2.95\%) cases. - Fig. 2 shows the types of swallowed FBs. The FB most commonly found in children was coins, while adults

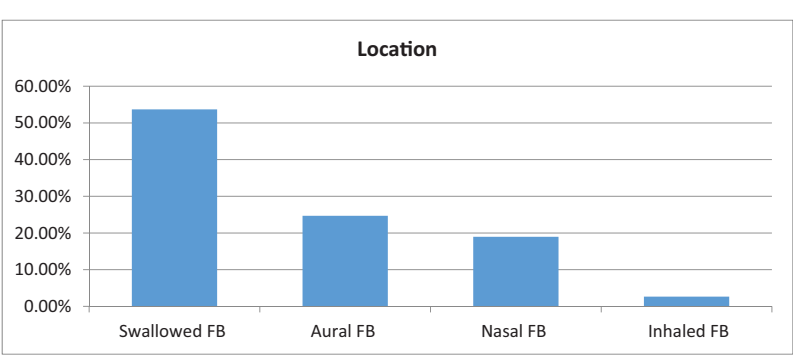

Fig. 1 Site of foreign bodies (FBs) impaction.

Table 1 Distribution of ENT foreign bodies by site, gender and age groups

\begin{tabular}{|l|l|l|l|l|l|l|l|l|l|}
\hline & \multicolumn{2}{|l|}{ Sex } & \multicolumn{2}{l|}{ Age } & Total \\
\cline { 2 - 9 } & Male & Female & $\mathbf{0 - 6}$ & $\mathbf{7 - 1 2}$ & $\mathbf{1 3 - 1 9}$ & $\mathbf{2 0 - 3 9}$ & $\mathbf{4 0 - 6 0}$ & $>\mathbf{6 0}$ & \\
\hline Swallowed FBs & 314 & 230 & 342 & 90 & 17 & 38 & 36 & 21 & 544 \\
& $57.7 \%$ & $42.3 \%$ & $62.9 \%$ & $16.5 \%$ & $3.13 \%$ & $6.99 \%$ & $6.62 \%$ & $3.86 \%$ & $100 \%$ \\
\hline Aural FBs & 160 & 90 & 81 & 100 & 15 & 34 & 19 & 1 & 250 \\
& $64 \%$ & $36 \%$ & $32.4 \%$ & $40 \%$ & $6 \%$ & $13.6 \%$ & $7.6 \%$ & $0.4 \%$ & $100 \%$ \\
\hline Nasal FBs & 87 & 105 & 44 & 94 & 22 & 31 & 1 & 0 & 192 \\
& $45.31 \%$ & $54.69 \%$ & $22.92 \%$ & $48.96 \%$ & $11.46 \%$ & $16.14 \%$ & $0.52 \%$ & & $100 \%$ \\
\hline Inhaled FBs & 11 & 16 & 20 & 3 & 2 & 0 & 1 & 1 & 27 \\
& $40.74 \%$ & $59.26 \%$ & $74.1 \%$ & $11.11 \%$ & $7.41 \%$ & & $3.69 \%$ & $3.69 \%$ & $100 \%$ \\
\hline
\end{tabular}

Abbreviations: ENT, ear, nose and throat; FB, foreign body. 
Table 2 Methods of removal ENT foreign bodies

\begin{tabular}{|l|l|l|l|l|}
\hline & $\begin{array}{l}\text { Removal } \\
\text { under LA }\end{array}$ & $\begin{array}{l}\text { Removal } \\
\text { under GA }\end{array}$ & $\begin{array}{l}\text { No FB } \\
\text { found }\end{array}$ & Total \\
\hline Swallowed FBs & 30 & 466 & 48 & 544 \\
& $5.52 \%$ & $85.66 \%$ & $8.82 \%$ & $100 \%$ \\
\hline Aural FBs & 216 & 34 & 0 & 250 \\
& $86.4 \%$ & $13.6 \%$ & & $100 \%$ \\
\hline Nasal FBs & 165 & 27 & 0 & 192 \\
& $85.94 \%$ & $14.06 \%$ & & $100 \%$ \\
\hline Inhaled FBs & 0 & 27 & 0 & 27 \\
& & $100 \%$ & & $100 \%$ \\
\hline
\end{tabular}

Abbreviations: ENT, ear, nose and throat; FB, foreign body; GA, general anesthesia; LA, local anesthesia.

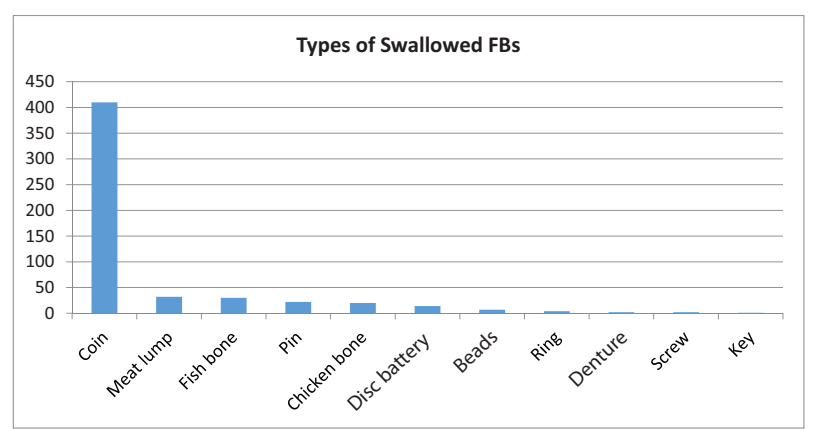

Fig. 2 Types of swallowed FBs.

presented more with fish bones and chicken bones, while elderly patients presented more with meat lumps and artificial dentures. The clinical features of these patients were mainly dysphagia for solids followed by throat pain, FB sensation inside the throat, odynophagia and pooling of saliva. The most common site of impaction was the hypopharynx, particularly at the cricopharyngeal sphincter, and it occurred in $370(68.01 \%)$ patients. The other sites of FB impaction were the esophagus in 96 (17.65\%) patients, and the oropharynx and oral cavity, both in $30(5.51 \%)$ patients.

Radiographs were performed when the FB could not be found to aid in the determination of the exact site of the FB. These were mainly plain X-ray neck lateral views, and, when necessary, chest and abdominal radiographs. About 450 (82.72\%) FBs were radio opaque, and 64 (11.76\%) FBs showed normal radiograph. In $30(5.52 \%)$ cases, no X-ray was performed, because the FB was visible to the naked eye, as it was located in the oropharynx/oral cavity.

Out of 544 swallowed FBs, 466 patients (with FBs in the hypopharynx and esophagus) had their FBs removed under GA via rigid endoscopy (hypopharyngoscopy/ esophagoscopy); 30 (5.52\%) cases (with FBs in the tonsil/oropharynx, valeculla, base of the tongue) were removed with or without LA by grasping forceps after the tongue had been depressed with a tongue depressor under proper illumination with a head lamp or with head mirrors that directed light to the oropharynx. A total of 48 (8.82\%) cases had no FBs removed in spite of positive history because of the descent of the FB or false sensation by the patient.
No complications were observed, but hospitalization for a few days for observation was needed in $6(1.1 \%)$ cases that had long-standing neglected coins or disc batteries.

\section{Inhaled Foreign Bodies}

A total of 27 cases presented with laryngo-tracheobronchial FBs, of which 11 (40.74\%) were male patients and 16 (59.26\%) were females. The highest incidence occurred among children $0-6$ years of age, who comprised $20(74.1 \%)$ cases. The most common inhaled FBs were beads ( 12 cases- $44.44 \%$ ), followed by pins ( 9 cases- $33.33 \%$ ), food particles ( 2 cases- $7.41 \%$ ), and other FBs (4 cases- 14.82\%). - Fig. 3 shows the types of inhaled FBs.

The patients presented with strong clinical suspicion of $\mathrm{FB}$ inhalation, with a history of coughing, choking, difficulty in breathing, along with the presence of clinical signs, like decreased air entry, wheezing, cyanosis, or crepitation. Chest $\mathrm{X}$-ray posteroanterior (PA) view showed either radio opaque FBs, obstructive emphysema, or collapse of the affected lung with mediastinal shift.

Rigid bronchoscopy was used to remove the inhaled FB in all cases (100\%). Pre-operatively, the most common complication of FB aspiration was stridor in 3 cases (11.11\%), followed by atelectasis in 2 cases (7.41\%), and recurrent pneumonia in 2 cases (7.41\%). No intraoperative or postoperative complications were encountered.

\section{Foreign Bodies in the Ear}

Out of the 250 patients who presented with FBs in their ears, 160 were males (64\%) and 90 were females (36\%). The highest incidence of ear FBs occurred in children 7-12 years of age, comprising 100 (40\%) cases. This was followed by children younger than 6 years of age, who comprised 81 (32.4\%) cases. Out of the FBs lodged in the ear, 165 (66\%) were in the right ear, and $85(34 \%)$ in the left ear. A total of $50(20 \%)$ patients had beads in their ears, followed by cotton tips and insects, which were found in $40(16 \%)$ cases, beans in $38(15.2 \%)$ cases, paper in $20(8 \%)$ cases, metallic FBs in 20 (8\%) cases, and other FBs in $42(16.8 \%)$ cases. - Fig. 4 shows the types of aural FBs.

Most of the patients with aural FBs (75\%) were asymptomatic at presentation, with history of verbal admission by the patient or incident witnessed by the caregiver. Other patients presented with otalgia, bleeding from the ear, otorrhea, tinnitus, hearing loss, a sense of ear fullness or symptoms of otitis media. All the FBs were seen on direct vision with or without otoscopic assistance. Regarding their locations, the most frequent

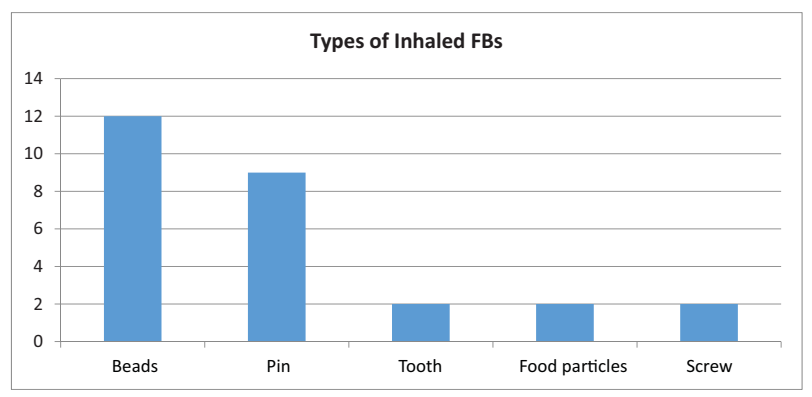

Fig. 3 Types of inhaled FBs. 
anatomical site of FB in the ear is the bony part of the external auditory canal. Previous attempts of removing the aural FBs had been made in 50 (20\%) patients before they presented to our hospital.

Of the 250 cases of FB in the ear, 216 (86.4\%) were removed in the OD or ENT emergency unit with or without LA by ear syringing, suctioning, forceps, probe, or fine hook. Only 34 (13.6\%) cases required removal under GA because these patients were uncooperative or due to impaction of the FB. Live insects were killed first by drowning in olive oil before eventual ear syringing.

Complications were reported in 30 (12\%) patients with FBs in their ears, especially in those with previous failed attempts at removal. Complications included the following: otitis externa (14 cases - 5.6\%), laceration/bruising of the external canal ( 8 cases $-3.2 \%$ ), tympanic membrane perforation ( 4 cases $-1.6 \%$ ), and acute otitis media ( 4 cases $-1.6 \%$ ).

\section{Foreign Bodies in the Nose}

Foreign bodies lodged in the nose were encountered in 192 patients, 87 (45.31\%) of which were males, and 105 (54.69\%) of which were females. A total of 44 (22.92\%) patients were younger than 6 years old, and 94 (48.96\%)had ages between 7 and 12 . A total of $155(80.7 \%)$ nasal FBs were in the right nasal cavity, and 37 (19.3\%) were in the left.

Beads were the most common type of FB found in the nose, constituting 91 (47.39\%) cases. This was followed by stones in 55 (28.65\%) cases, beans or seeds in 21 (10.94\%) cases, and other FBs in 25 (13.02\%) cases. - Fig. 5 describes the types of nasal FBs.

The patients presented with a history of introduction of the FB by themselves or with a history of guardians who saw the FB in the nose, local pain, unilateral offensive nasal discharge in cases of neglected FBs, epistaxis, and other symptoms (rhinitis, nasal obstruction, sensation of swelling). Most of the FBs were seen on direct vision. The nasal endoscope was employed to locate deep-seated nasal FBs. Previous attempts of removing nasal FBs had been made in 20 (10.42\%) patients before they presented to our hospital.

Of 192 cases of FB in the nose, 165 (85.94\%) were removed in the OD or ENT emergency unit with or without LA under direct vision by hook, forceps and by suctioning. Only 27 (14.06\%) cases required removal under GA, because these patients were uncooperative or due to a deep-seated FB.

Only 14 patients (7.29\% of all cases) with nasal FBs had complications: acute rhinosinusitis (6 cases $-3.13 \%$ ), epis-

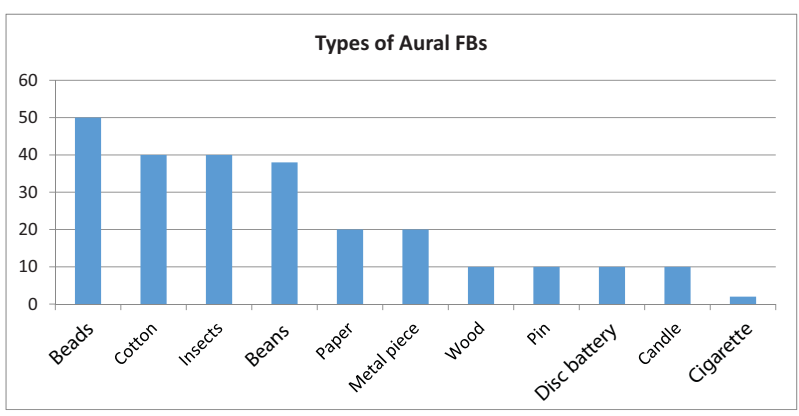

Fig. 4 Types of aural FBs. taxis ( 3 cases - 1.56\%), laceration ( 2 cases $-1.04 \%$ ), rhinolith formation ( 2 cases $-1.04 \%$ ) and ulceration of the nasal septum ( 1 case $-0.52 \%$ ).

\section{Discussion}

The present study considered patients examined for ENT FBs in the OD and in the ENT emergency unit in a tertiary referral hospital for 2 consecutive years (from July 2014 to June 2016). The 1,013 cases of ENT FBs accounted for $30 \%$ of all patients examined in the ENT emergency services during this period. According to the literature, FBs account for $\sim 11 \%$ of the cases observed in ENT emergency services. ${ }^{2-4}$ Ear, nose and throat FB injuries represent an emerging problem in the population, especially in the pediatric age group, because of their human and social costs. ${ }^{10,11}$ In the present study, FBs represented the most common ENT emergencies; this is similar to other studies, which also recorded FBs to be the most common ENT emergencies. ${ }^{12}$

In the present study, FBs were more prevalent in children: $48.07 \%$ of the patients aged 6 years or younger, and $28.33 \%$ of the patients aged 7-12 years presented with FBs. Male individuals were the majority by a slight difference (56.47\%).These findings are in agreement with the literature and with reports of FBs being more common in children around 6 years of age. ${ }^{2,13,14}$ Several factors can be responsible for the age distribution; an analysis of the literature reveals that the mouthing activity of children appears to be the most important factor. Insertion of FBs by the little children may also be precipitated by boredom and frustration, or they also may be mimicking the unhealthy habits of ear and nose picking by adults. ${ }^{13,15-17}$ Patients with psychological problems are prone to FB insertion; suitable measures should be taken to prevent them from putting FBs in their nose or ears. ${ }^{18}$

Many studies in the literature reported aural FBs as the most prevalent. ${ }^{4,19}$ In the present study, swallowed FBs were the most common (53.7\%), followed by aural FBs (24.68\%), nasal FBs (18.95\%), and finally FB inhalation (2.67\%). In our study, the most common site of FB lodgment was the throat, which is in corroboration with other studies that reported similar rates of occurrence of ENT FBs. ${ }^{20,21}$

The key to successful removal is immobilization. ${ }^{4}$ Quick atraumatic removal of FBs is a real challenge for ENT physicians. Therapeutic success depends on several factors, but there is no strong evidence to indicate that one specific removal method should be performed over others. ${ }^{22}$ It is known, however, that the permanence of FBs in ENT cavities

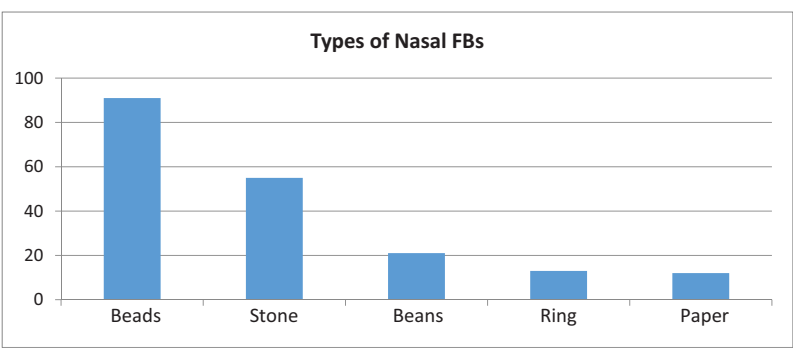

Fig. 5 Types of nasal FBs. 
for over 72 hours and repeated attempts to remove the FB increase the risk of complications. ${ }^{2,3,23}$ In our study, the FB was removed with or without LA in $40.57 \%$ of the patients; $54.69 \%$ of the patients required removal under $\mathrm{GA}$; and no FB was found in $4.74 \%$ of the patients, in spite of positive history. In our study, the need for GA to remove the FBs was higher than the rates reported in the literature (around 30\%). $3,6,24$

A study by Barreto and Holinger in 2005 mentioned that, among the upper digestive tract FB cases, fish bone was the most common $(70.5 \%)$, followed by coin in the esophagus (6.63\%), artificial denture impaction in the esophagus (1.89\%), and meat bone impaction in the esophagus (1.49\%). ${ }^{25}$ In our study, the most commonly ingested FBs were coins (75.37\%), followed by meat lumps (5.88\%) and fish bones (5.51\%). Since coins are used as currency, toddlers and children have easy access to them, and accidentally swallow them. The most common site of lodgment was the cricopharynx, and that correlates with other studies. ${ }^{19,26}$ Foreign body ingestion is common among children, but it is frequently observed among intellectually disabled or mentally ill adults as well. ${ }^{27}$

A total of $85.66 \%$ (466 cases) of the patients with swallowed FBs required GA for removal. A total $5.52 \%$ (30 cases) of the patients had the FB removed with or without LA, and $8.82 \%$ (48 cases) of the cases had no FB removed in spite of positive history, because of the descent of the FB or false sensation by the patient. No complications were observed, but hospitalization for a few days for observation was needed in $6(1.1 \%)$ cases that had long-standing neglected coins or disc batteries. Disc batteries may release small amounts of chemicals and voltage that may lead to alkaline chemical burns, necrosis, or even perforation. ${ }^{28}$ Batteries should be removed as soon as possible to prevent these complications.

Inhaled FBs were recorded in $2.67 \%$ of the cases in our study. In most series, inhaled FBs are generally food particles, and the child is eating and playing at the same time the accident happens. ${ }^{25,29}$ In the present study FB most commonly reported were beads ( 12 cases $-44.44 \%$ ), followed by pins ( 9 cases $-33.33 \%$ ) and food particles ( 2 cases $-7.41 \%$ ).

Barretto and Holinger reported a $56.6 \%$ prevalence in 1-3 year-old children, and explained it by the fact that they are learning to walk and have a tendency to put objects in their mouths. ${ }^{25}$ Lescanne et al, in 1997 , found that a $1.8: 1$ sex ratio in favor of males is in agreement with the literature. They found that children under 3 years old are most often involved, and the FB location is usually bronchial. They asked the following questions: Is their higher level of activity what puts boys at a greater risk? Or is it a difference in pharyngo-laryngeal junction maturity ${ }^{29}$ In our series, children aged 6 years or younger constituted $74 \%$ of the cases ( 8 cases [29.62\%] were of children 3 years old or younger); in contrast to the previous study, the sex ratio was $1.45: 1$ in favor of females. The differences from other series in the literature regarding the type of FBs, age and sex of the patients may be caused by the fact that only selected cases were managed in our department, and most of inhaled FBs presented to our hospital have been managed in the Cardiothoracic Department.

All cases in our study were managed using rigid bronchoscopy. As stated in literature, rigid bronchoscopy remains the attitude of choice. It should be performed under GA and spontaneous ventilation by an experienced operator and an appropriately trained anaesthetist. ${ }^{30}$ In our study, the most common preoperative complication of FB aspiration was stridor, which was observed in 3 cases (11.11\%). No intraoperative or post-operative complications were encountered. Naragund et al (2014) reported that the most common complication of FB aspiration encountered pre-operatively was persistent pneumonia, intraoperative bleeding from the site of the FB, and post-operative bronchospasm. ${ }^{31}$

Cases with predisposing ear problems, like itching, pain, heaviness, discomfort, and hearing impairment may push children and adults to probe the ear with some object, leading to FB impaction, as well as injuries. ${ }^{13,32}$ In our study, children were the most affected age group. The FBs were generally found in males (64\%) more than in females (36\%). The most common FBs in children were beads, insects and disc batteries, while cotton, paper and beans were the most common in adults.

In 1998, Ainsley and Cunningham reported that the most common FBs in the ear are beads, plastic toys, pebbles, popcorn kernels and insects. Thirty percent of the patients in their study required GA to facilitate the removal of an ear FB. ${ }^{6}$ The most common ear FBs in our study were beads, followed by cotton tips, insects and beans.

Most aural FBs can be removed in the office; insects in ears should be killed by instillation of oil into the external auditory canal. Uncooperative children and some adults require GA to remove their $\mathrm{FBs}$ and to assess the condition of the tympanic membrane using the microscope. ${ }^{33,34}$ In our study, most ear FBs (86.4\%) were removed in the office, and live insects were killed first by drowning them in oil before eventual ear syringing. Only $13.6 \%$ of patients required GA to facilitate the removal of ear FBs. The decisive factors were patient age, cooperation, and the degree of impaction.

Complications due to the presence of an FB or due to the extraction were uncommon; the complications were recorded in $12 \%$ of the cases, and that is in agreement with a study by Al-Juboori, ${ }^{35}$ who recorded a $12.5 \%$ complication rate, and in contrast with a study by Singh et al, who recorded a $77 \%$ complication rate. ${ }^{36}$ Complications were reported more in patients with previous failed attempts at removal.

The common nasal FBs mentioned in the literature include beads, buttons, toy parts, pebbles, candle wax, food, paper, cloth, and disc batteries. Most nasal FBs can be removed with direct visualization using a forceps, curved hooks, or suction catheters. $^{37,38}$ In the present study, common nasal FBs included beads, stones, and beans.

The FBs observed in the nose in our study were mostly found in children younger than 12 years old (71.88\%).These findings are in agreement with the literature. ${ }^{2,8,21,26}$ Contrary to FBs in the ear, females (105 cases - 54.69\%) had a higher incidence than males ( 87 cases $-45.31 \%$ ).

As such, the physician should have several techniques for removing the different types of FB. Numerous techniques have been well-described. ${ }^{39}$ The methods used most successfully for removal of $85.94 \%$ ( 165 cases) of nasal FBs in our OPD and ENT Emergency unit with or without LA by direct visualization include the use of forceps, hooks and suctioning. Only $14.06 \%$ 
(27 cases) of nasal foreign bodies were removed under GA using a nasal endoscope; this was performed in uncooperative children and for FBs located far posteriorly in the nasal cavity.

The complications that occurred were usually related to repeated attempts at removal. ${ }^{40}$ Only $7.29 \%$ of the patients (14 cases) with nasal foreign bodies were recorded in our study to had complications.

\section{Conclusion}

Foreign bodies in the ears, nose or throat are a common occurrence in otorhinolaryngology emergency services. Children are the most affected age group. The most common site of FB lodgment is in the throat. Ear, nose and throat FBs need to be properly managed to avoid complications.

\section{References}

1 Thompson SK, Wein RO, Dutcher PO. External auditory canal foreign body removal: management practices and outcomes. Laryngoscope 2003;113(11):1912-1915

2 Figueiredo RR, Azevedo AA, Kós AO, Tomita S. Complications of ent foreign bodies: a retrospective study. Rev Bras Otorrinolaringol (Engl Ed) 2008;74(01):7-15

3 Silva BSR, Souza LO, Camera MG, Tamiso AGB, Castanheira LVR. Foreign bodies in otorhinolaryngology: a study of 128 cases. Int Arch Otorhinolaryngol 2009;13(04):394-399

4 Bressler K, Shelton C. Ear foreign-body removal: a review of 98 consecutive cases. Laryngoscope 1993;103(4 Pt 1):367-370

5 Yuca K, Yuca SA, Caksen H. Aural live foreign bodies in children. J Emerg Med 2003;25(01):102-104

6 Ansley JF, Cunningham MJ. Treatment of aural foreign bodies in children. Pediatrics 1998;101(4 Pt 1):638-641

7 Ologe FE, Dunmade AD, Afolabi OA. Aural foreign bodies in children. Indian J Pediatr 2007;74(08):755-758

8 Shrestha I, Shrestha BL, Amatya RCM. Analysis of ear, nose and throat foreign bodies in dhulikhel hospital. Kathmandu Univ Med J (KUMJ) 2012;10(38):4-8(KUMJ)

9 Pecorari G, Tavormina P, Riva G, Landolfo V, Raimondo L, Garzaro M. Ear, nose and throat foreign bodies: the experience of the Pediatric Hospital of Turin. J Paediatr Child Health 2014;50(12):978-984

10 Gregori D, Scarinzi C, Berchialla P, et al; ESFBI Study Group. The cost of foreign body injuries in the upper aero-digestive tract: need for a change from a clinical to a public health perspective? Int J Pediatr Otorhinolaryngol 2007;71(09):1391-1398

11 Harlan LC, Harlan WR, Parsons PE. The economic impact of injuries: a major source of medical costs. Am J Public Health 1990;80(04):453-459

12 Patigaroo SA, Ahmad R, Khan MA, Afzal A, Khan MA. ENT Emergencies- An Experience. Ind. J. Sci. Res. and Tech. 2013;1(03):62-65

13 Mukherjee A, Haldar D, Dutta S, Dutta M, Saha J, Sinha R. Ear, nose and throat foreign bodies in children: a search for socio-demographic correlates. Int J Pediatr Otorhinolaryngol 2011;75(04):510-512

14 Tiago RS, Salgado DC, Corrêa JP, Pio MR, Lambert EE. Foreign body in ear, nose and oropharynx: experience from a tertiary hospital. Rev Bras Otorrinolaringol (Engl Ed) 2006;72(02):177-181

15 Iwata S, Mishima Y, Nishimura T, et al. 615 cases with foreign bodies in the esophagus and bronchotracheal tree during the last 10 years/as statistical analysis gathered from the departments of ENT of 7 universities in the Tokai area in Japan. J. Jpn. Bronchoesophagol. 1996;47:510-525

16 Tulve NS, Suggs JC, McCurdy T, Cohen Hubal EA, Moya J. Frequency of mouthing behavior in young children. J Expo Anal Environ Epidemiol 2002;12(04):259-264
17 Juberg DR, Alfano K, Coughlin RJ, Thompson KM. An observational study of object mouthing behavior by young children. Pediatrics 2001;107(01):135-142

18 Bakhshaee M, Hebrani P, Shams M, Salehi M, Ghaffari A, Rajati M. Psychological status in children with ear and nose foreign body insertion. Int J Pediatr Otorhinolaryngol 2017;92:103-107

19 Endican S, Garap JP, Dubey SP. Ear, nose and throat foreign bodies in Melanesian children: an analysis of 1037 cases. Int J Pediatr Otorhinolaryngol 2006;70(09):1539-1545

20 Phillipps JJ, Patel P. Swallowed foreign bodies. J Laryngol Otol 1988;102(03):235-241

21 Sarkar S, Roychoudhury A, Roychaudhuri BK. Foreign bodies in ENT in a teaching hospital in Eastern India. Indian J Otolaryngol Head Neck Surg 2010;62(02):118-120

22 Heim SW, Maughan KL. Foreign bodies in the ear, nose, and throat. Am Fam Physician 2007;76(08):1185-1189

23 Balbani AP, Sanchez TG, Butugan O, et al. Ear and nose foreign body removal in children. Int J Pediatr Otorhinolaryngol 1998; 46(1-2):37-42

24 Ikino CMY, D'Antonio WEPA, Balbani APS, Sanchez TG, Butugan O. Análise dos Atendimentos para Retirada de Corpos estranhos de Ouvido e Nariz em Crianças. Rev Bras Otorrinolaringol 1998; 64(04):379-383

25 Barretto RL, Holinger LD. Foreign bodies of the airway and esophagus. In: Cummings, et al., editors. Pediatric otolaryngology head and neck surgery. Elsevier Mosby; 2005; 4343-8

26 Ray R, Dutta M, Mukherjee M, Gayen GC. Foreign body in ear, nose and throat: experience in a tertiary hospital. Indian J Otolaryngol Head Neck Surg 2014;66(01):13-16

27 Sanowski RA. Foreign body extraction in the gastrointestinal tract. In: Sivak MV, editor. Gastroenterological endoscopy. Philadelphia: W. B. Saunders Co; 1987. pp. 321-331.

28 Palmer O, Natarajan B, Johnstone A, Sheikh S. Button battery in the nose-an unusual foreign body. J Laryngol Otol 1994;108(10): 871-872

29 Lescanne E, Soin C, Lesage V, Mercier C, Ployet MJ. Corps étrangers laryngo-tracheo-bronchiques. Encyclopédie Médico- Chirurgicale (Elsevier, Paris) ORL; 1997;20-730-A-10:1-10.

30 Rizk H, Rassi S. Foreign body inhalation in the pediatric population: lessons learned from 106 cases. Eur Ann Otorhinolaryngol Head Neck Dis 2011;128(04):169-174

31 Naragund AI, Mudhol RS, Harugop AS, Patil PH, Hajare PS, Metgudmath VV. Tracheo-bronchial foreign body aspiration in children: a one year descriptive study. Indian J Otolaryngol Head Neck Surg 2014; 66(01, Suppl 1):180-185

32 Mishra A, Shukla GK, Bhatia N. Aural foreign bodies. Indian J Pediatr 2000;67(04):267-269

33 Ghosh P. Foreign bodies in ear, nose and throat (Predictions and management). Indian J Otolaryngol Head Neck Surg 1999; 51(Suppl 1):2-5

34 Leffler S, Cheney P, Tandberg D. Chemical immobilization and killing of intra-aural roaches: an in vitro comparative study. Ann Emerg Med 1993;22(12):1795-1798

35 Al-Juboori AN. Aural foreign bodies: descriptive study of 224 patients in Al-fallujah general hospital, iraq. Int J Otolaryngol 2013;2013:401289

36 Singh GB, Sidhu TS, Sharma A, Dhawan R, Jha SK, Singh N. Management of aural foreign body: an evaluative study in 738 consecutive cases. Am J Otolaryngol 2007;28(02):87-90

37 Chan TC, Ufberg J, Harrigan RA, Vilke GM. Nasal foreign body removal. J Emerg Med 2004;26(04):441-445

38 Fox JR. Fogarty catheter removal of nasal foreign bodies. Ann Emerg Med 1980;9(01):37-38

39 Kadish HA, Corneli HM. Removal of nasal foreign bodies in the pediatric population. Am J Emerg Med 1997;15(01):54-56

40 Tong MC, Ying SY, van Hasselt CA. Nasal foreign bodies in children. Int J Pediatr Otorhinolaryngol 1996;35(03):207-211 